\title{
How Should We View the Results of the Multicenter Selective Lymphadenectomy Trial-1 (MSLT-1)?
}

\author{
Merrick I. Ross, MD and Jeffrey E. Gershenwald, MD \\ Department of Surgical Oncology, Unit 444, The University of Texas M. D. Anderson Cancer Center, 1515 Holcombe Blvd, \\ Houston, TX 77030, USA
}

Prior to the release of the initial findings of the Multicenter Selective Lymphadenectomy Trial-1 (MSLT-1), SLN biopsy had already been generally embraced by the worldwide surgical community as a valuable staging tool and as a minimally invasive way to accurately identify patients with clinically occult regional lymph node metastases. The application of regional lymph node dissection only for patients with positive SLNs-termed "selective lymphadenectomy"-effectively minimizes the development of clinical nodal disease and spares node-negative patients the morbidity of a formal lymph node dissection. This management strategy has been promoted as a rational alternative to the two prior popularized approaches of elective lymph node dissection (ELND) or nodal observation. At the same time, a relatively vocal minority argued that a survival advantage from SLN biopsy should be demonstrated before the routine use of this procedure was endorsed.

In the fall of 2006, the highly anticipated results of the third of five planned interim analyses of the MSLT-1 were published. ${ }^{1}$ This trial was undertaken to compare the outcomes of patients with newly diagnosed primary cutaneous melanoma treated with either WLE plus observation of the regional nodes followed by regional lymphadenectomy if nodal metastases became clinically apparent (the observa-

Published online: January 29, 2008.

Address correspondence and reprint requests to: Jeffrey E. Gershenwald, MD; E-mail: jgershen@mdanderson.org

Published by Springer Science+Business Media, LLC @ 2008 The Society of Surgical Oncology, Inc. tion group) or WLE plus SLN biopsy (the SLN group) and (1) immediate regional lymphadenectomy if a SLN was positive (ie, selective lymphadenectomy) or (2) observation if the SLN was negative. The primary goal was to assess the survival impact of SLN biopsy; the trial also addressed several other questions regarding the use of SLN biopsy in the initial management of patients with melanoma.

This interim analysis of the MSLT $-1^{1}$ confirms previous reports that SLN status is the most powerful independent predictor of survival, but does not provide a definitive answer on whether SLN biopsy provides a survival advantage. With a median followup of 5 years, this analysis showed a 3\%, non-significant survival advantage for patients in the SLN biopsy group. Careful consideration of the trial data suggests that this lack of overall survival benefit from SLN biopsy is not surprising. In order to fully understand the results of this interim MSLT-1 analysis, reflection upon previous randomized ELND trials is instructive.

A significant body of evidence now exists related to the survival impact of treating microscopic nodal disease in patients with melanoma. In two randomized trials in melanoma patients at high risk for clinically occult nodal disease, ${ }^{2,3}$ ELND was compared with nodal observation followed by delayed lymph node dissection if palpable nodal disease developed. In both trials, there was a trend toward improved survival in the ELND group, but this was not significant. Critics concluded from these trials that early treatment of nodal metastases has little impact on disease progression. However, others suggested that because only the $20 \%$ of patients who 
harbored clinically occult nodal disease could potentially benefit from ELND, the trials were underpowered to detect a survival difference. In contrast, analyses limited to patients with nodal disease have shown a survival benefit from early lymph node dissection. The post-hoc analysis of the World Health Organization truncal melanoma randomized ELND trial showed a significantly higher 5-year survival rate for patients with microscopically positive lymph nodes who underwent ELND at the time of wide local excision (WLE) than for those who underwent delayed lymph node dissection after developing clinical disease $(48 \%$ vs. $27 \%, P=.04) .{ }^{3}$ The results of a multi-center retrospective trial from Germany revealed that completion lymph node dissection following a positive SLN biopsy improved survival compared with delayed dissection of clinically apparent lymph node metastases $(63 \%$ versus $50 \%$ at 5 -years). ${ }^{4}$ These data call into question the practice of delaying lymphadenectomy until palpable disease develops in patients with clinically negative lymph nodes.

Similar trial design limitations exist in MSLT-1. First, only $19 \%$ of the patients in the SLN group (i.e., those with a positive SLN at the time of biopsy) could potentially benefit from early therapeutic lymph node dissection. If one assumes, based on observations from the aforementioned trials, that approximately $15 \%-20 \%$ of the microscopic node positive patients actually benefit from an early dissection, a survival advantage of no more than $3 \%-4 \%$ (i.e., $15-20 \%$ of $19 \%$ ) would be expected for the overall SLN biopsy group compared to the overall observation group. The trial did not have sufficient power to detect such a survival benefit. However, the two above-noted ELND trials showed that with increasing follow-up, survival differences increased as more events occurred in the observation group than in the immediate ELND group. ${ }^{2,3}$ It therefore seems appropriate to speculate that greater survival differences may emerge with longer follow-up of the MSLT-1.

An additional planned analysis from the MSLT-1 trial compared the patients who had positive SLNs and underwent immediate lymphadenectomy versus the patients in the observation group who later developed palpable nodal disease. The results from this secondary analysis are particularly noteworthy: there was a significant progression to more advanced nodal disease in the nodal observation group, and there was a significant survival advantage $(20 \%)$ for the SLN-positive patients who underwent immediate lymphadenectomy. These data corroborate the earlier findings from the World Health Organization and
German studies described above and demonstrate that left intact, micrometastases can grow and be associated with a worse prognosis. ${ }^{3,4}$ Such increased nodal burden may also represent a source of distant dissemination. Thus, this secondary analysis shows that early treatment of nodal disease can favorably alter the natural history of melanoma.

Opponents of the SLN approach have criticized this subset analysis and maintain that this survival comparison is invalid because the patients were not randomly assigned to these subgroups and the underlying biology of patients with microscopic lymph node involvement may not necessarily be the same as for patients who developed palpable nodal disease. Others have suggested that the reason for the improved survival observed in the SLN-positive group is that the small volume of disease identified in some of the patients was clinically irrelevant-i.e., that this nodal disease would never have progressed and that the positive SLNs in these patients should be considered "falsely positive." Recently, a review ${ }^{5}$ and accompanying editorial ${ }^{6}$ - both focusing on the MSLT-1 - were published in Nature Clinical Practice Oncology. These articles suggested that survival differences thus far observed among the various nodepositive groups in the MSLT-1 could be explained by differences in their constellation of prognostic factors.

While such criticisms should be considered, it is also logical to conclude, based on the MSLT-1 data, that the patients in the two subgroups were similar and that the microscopic metastases in SLNs would most likely have progressed to palpable disease if left intact, for the following five reasons. (1) Since this was a well-designed and appropriately stratified prospective randomized trial, the incidence of microscopic nodal disease in the two treatment groups should have been identical. (2) The percentage of SLN-group patients with nodal disease (discovered at SLN biopsy or as false-negative events during subsequent follow-up and observation) is nearly identical to the percentage of observation-group patients who have thus far developed palpable nodal disease and is predicted to be identical by 10 years of follow-up (Fig. 1) ${ }^{7}$ (3)The mean number of positive nodes was 1.6 in the SLN-positive group, 3.6 in the nodal observation group, and 4.3 in the false-negative SLN group, (ie, greater tumor burden in the two patients groups in whom regional disease was not removed early). (4) If a significant proportion of the positive SLNs were not clinically relevant, the rate of false-negative findings of SLN biopsy would have been higher. (5) The assertion that the node-positive 


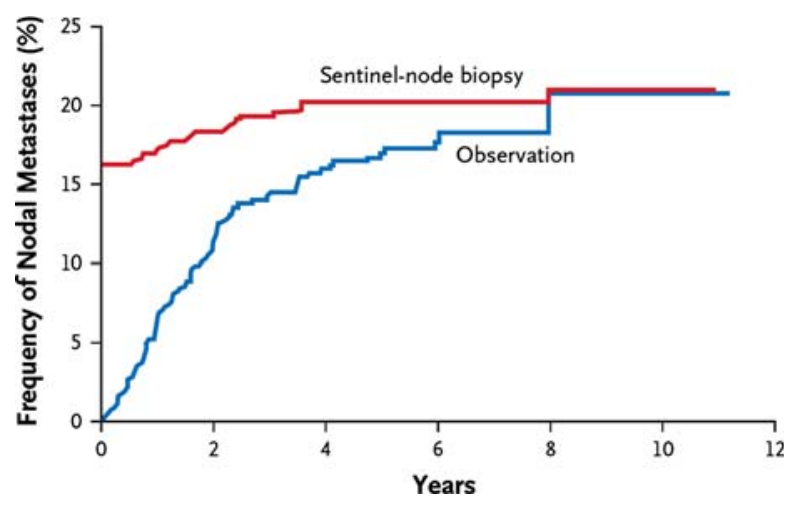

FIG. 1. Cumulative incidence of regional node metastasis in the observation group and the biopsy group in MSLT-1. From Morton DL, Cochran AJ, Thompson JF: Authors' reply to a letter to the editor re: Sentinel-Node Biopsy in Melanoma. N Engl J Med 356:418-9; author reply 419-21, 2007. Copyright (C) 2007 Massachusetts Medical Society. All rights reserved.

groups-i.e., following nodal observation, positive SLN biopsy, or false-negative SLN biopsy-are prognostically different is unfounded and erroneous. As reported in the MSLT-1 study, the median primary tumor thickness and ulceration status of these groups were essentially the same and not statistically different. ${ }^{1}$ Therefore, the most likely - and arguably the simplest - explanation for the observed survival differences between these node-positive groups is that removal of positive SLNs followed by completion lymph node dissection minimized the progression of nodal disease and the associated increased risk of distant dissemination. Longer follow-up of these patients is warranted to determine if over time the incidence of palpable nodal recurrence among patients randomized to nodal observation will continue to be higher than the incidence of additional falsenegative events in the SLN-negative group. If these future events follow the patterns observed in the two aforementioned ELND trials, ${ }^{2,3}$ there might be more recurrences in the nodal observation arm over time than in the SLN biopsy arm.

An additional argument promulgated against the routine use of SLN biopsy has been that early treatment of the regional nodal basin could increase the risk of in-transit disease. This concern has been laid to rest by multiple recent compelling evidence-based studies $^{8-11}$, including the MSLT $-1 .{ }^{1}$ In one study of approximately 3400 patients with melanoma treated with WLE alone, WLE plus ELND, or WLE plus SLN biopsy, the incidence of in-transit disease was $4.9 \%$ in the WLE-alone group and $4.5 \%$ in the WLE-plusSLN-biopsy group, and a multivariate analysis indicated that predictors of in-transit disease included SLN status, tumor thickness, and ulceration, but not type of procedure performed. ${ }^{9}$ In other words, intransit metastasis was related to tumor biology, not the type of surgical procedure. Moreover, recent results from MSLT-I show no difference in the rate of intransit metastasis between patients in the WLE-alone group and those in the WLE-plus-SLN-biopsy group. ${ }^{1}$

Some groups have evaluated less invasive methods of lymph node evaluation, such as ultrasound. SLN biopsy is typically well tolerated, especially in comparison with ELND; it contributes little additional morbidity to WLE. ${ }^{12}$ However, at present, an experienced sonographer is able to identify only those SLN metastases that have reached a minimum diameter of approximately $4 \mathrm{~mm}$ to $5 \mathrm{~mm}$, which represents a significant burden of disease that itself is associated with a relatively high rate of future distant disease. Contrast this minimum threshold for detection to our own institutional experience, in which the median largest SLN metastatic focus was only $1 \mathrm{~mm}$ for patients who had a positive SLN biopsy between 2000 and 2003; fewer than one in five patients had a largest SLN metastatic focus $>4 \mathrm{~mm}$. Such limited sensitivity in detecting SLN disease argues against the routine use of sonography as the sole surveillance tool for early detection of clinically occult regional nodal disease.

Even in the absence of a clear overall survival advantage conferred by SLN biopsy, the routine use of this approach is justified in patients with primary melanoma at risk for regional metastases as it offers accurate staging information and enhanced regional disease control with little additional morbidity beyond what is normally experienced with WLE. Furthermore, the collective evidence from the MSLT-1, previous reported ELND trials, and a large retrospective multicenter trial together support the view that survival is improved for the subset of patients with microscopic nodal disease. Until a primary tumor marker or profile is identified that is a more reliable indicator of outcome and natural history, SLN biopsy should continue to be offered as a minimally invasive method of achieving accurate lymph node staging, achieving durable regional disease control, and optimizing the chance for cure in newly diagnosed patients with stage I and II melanoma.

\section{REFERENCES}

1. Morton DL, Thompson JF, Cochran AJ, et al. Sentinel-node biopsy or nodal observation in melanoma. $N$ Engl J Med 2006; 355:1307-17.

2. Balch C, Soong S, Ross M, et al. Long-term results of a multiinstitutional randomized trial comparing prognostic factors and surgical results for intermediate thickness melanomas (1.0 
to $4.0 \mathrm{~mm})$. Intergroup Melanoma Surgical Trial. Ann Surg Oncol 2000; 7:87-97.

3. Cascinelli N, Morabito A, Santinami M, et al. Immediate or delayed dissection of regional nodes in patients with melanoma of the trunk: a randomised trial. WHO Melanoma Programme. Lancet 1998; 351:793-6.

4. Kretschmer L, Hilgers R, Mohrle M, et al. Patients with lymphatic metastasis of cutaneous malignant melanoma benefit from sentinel lymphonodectomy and early excision of their nodal disease. European Journal of Cancer 2004; 40:212-8.

5. Thomas JM. Prognostic false-positivity of the sentinel node in melanoma. Nat Clin Pract Oncol 2008; 5:18-23.

6. Rosenberg SA. Why perform sentinel-lymph-node biopsy in patients with melanoma?. Nat Clin Pract Oncol 2008; 5:1.

7. Morton DL, Cochran AJ, Thompson JF: Authors' reply to a letter to the editor re: Sentinel-Node Biopsy in Melanoma. N Engl J Med 356:418-9; author reply 419-21, 2007.
8. Pawlik TM, Ross MI, Johnson MM, et al. Predictors and natural history of in-transit melanoma after sentinel lymphadenectomy. Ann Surg Oncol 2005; 12:587-96.

9. Pawlik TM, Ross MI, Thompson JF, et al. The risk of intransit melanoma metastasis depends on tumor biology and not the surgical approach to regional lymph nodes. J Clin Oncol 2005; 23:4588-90.

10. Kang JC, Wanek LA, Essner R, et al. Sentinel lymphadenectomy does not increase the incidence of in-transit metastases in primary melanoma. J Clin Oncol 2005; 23:4764-70.

11. van Poll D, Thompson JF, Colman MH, et al. A sentinel node biopsy does not increase the incidence of in-transit metastasis in patients with primary cutaneous melanoma. Ann Surg Oncol 2005; 12:597-608.

12. Wrightson WR, Wong SL, Edwards MJ, et al. Complications associated with sentinel lymph node biopsy for melanoma. Ann Surg Oncol 2003; 10:676-80. 